\title{
Method of X-ray residual stress measurement for phase transformed welds
}

\author{
Akihiro Tsuji • Shigetaka Okano • Masahito Mochizuki
}

Received: 29 October 2013 / Accepted: 11 February 2015 / Published online: 26 February 2015

(C) The Author(s) 2015. This article is published with open access at Springerlink.com

\begin{abstract}
In this study, we attempted to evaluate the residual stress distribution in welds accompanied by phase transformation using X-ray stress measurement. First, relationship between the phase transformation behavior and the residual stress of welded specimens with different phase transformation was discussed. In the specimen with martensite in the weld, reduction in tensile stress due to martensite transformation shown in residual stress distribution followed conventional behavior. However, in the specimen with bainitic ferrite in the weld, the residual stress in the transverse direction was almost the same as the residual stress in the longitudinal direction, and the residual stress did not follow conventional behavior. Next, X-ray elastic constant in the weld was measured, and then the residual stress was reevaluated. In the specimen with bainitic ferrite in the weld, X-ray elastic constant had anisotropy, and the reevaluated residual stress followed conventional behavior. In conclusion, it was shown that depending on the phase transformation behavior, it is difficult to use Xray elastic constant estimated by Kröner model; however, if we use the measured value, we are able to evaluate the residual stress more accurately.
\end{abstract}

Keywords Welding $\cdot$ Residual stresses $\cdot$ Transformation . $\mathrm{X}$-rays $\cdot$ Stress analysis $\cdot$ Measurement

Doc. IIW-2544, recommended for publication by Commission X "Structural Performances of Welded Joints - Fracture Avoidance".

A. Tsuji $(\bowtie) \cdot$ S. Okano $\cdot$ M. Mochizuki

Graduate School of Engineering, Osaka University, 2-1 Yamada-oka,

Suita, Osaka 565-0871, Japan

e-mail: akihiro_tsuji@mapse.eng.osaka-u.ac.jp

\section{Introduction}

It is well known that the residual stress from the welding process affects the various fracture strengths of a welded structure [1]. Therefore, evaluating the weld residual stress accurately is one of the most important technical issues for ensuring structural reliability. To evaluate the weld residual stress, various methods have been proposed, for example totally destructive methods such as the sectioning method [2], semidestructive methods such as the center-hole method [3], nondestructive methods such as the diffraction method [4], and numerical analysis using computer simulations. X-ray stress measurement is superior among the nondestructive and highresolution stress measuring methods for material surfaces that are prone to damage and cracking initiation. On the other hand, it has been thought that the application of X-ray stress measurement to the weld is difficult with respect to measurement accuracy and reliability because of the large grains and texture in the weld. However, due to the proactive approach in recent years, much knowledge regarding the application of Xray stress measurement to welds has been accumulated [5, 6].

Additionally, in structural steel welds, complex residual stress fields are formed due to phase transformations that occur according to the thermal cycles. Hardening due to microstructural changes accompanied by phase transformation raises the yield stress, and expansion due to martensitic transformation in relatively low temperatures reduces the tensile residual stress. Although the analytical evaluation of weld residual stress accompanied by phase transformation has been attempted by relating these residual stress behaviors with the cooling rate and modeling them $[7,8]$, the phase transformation behavior is affected by various materials science factors. Therefore, it is not always realistic to accurately evaluate the weld residual stress using only an analytical approach. So, it would be significant if we were able to evaluate complex residual stress distributions in welds accompanied by phase 
transformation in detail through an experimental approach using the nondestructive technique represented by X-ray stress measurement.

In this study, we used specimens with different phase transformation and evaluated in detail the residual stress distribution using X-ray stress measurement, focusing on the effect of phase transformation on the residual stress distribution. Moreover, we discussed the X-ray elastic constant on weld with phase transformation.

\section{X-ray stress measurement of different phase transformed welds}

\subsection{Material and welding conditions}

The material tested was rolled steel for welded structures (SM490YB). As shown in Fig. 1, the specimens were thin plates with a size of $100 \mathrm{~mm} \times 150 \mathrm{~mm} \times 6 \mathrm{~mm}$. Before welding, the specimens were heat treated at $873 \mathrm{~K}$ for $6 \mathrm{~h}$. After heat treatment, the surface oxide was scraped off with abrasive paper, and electrolytic polishing was performed to eliminate the initial residual stress, which was less than $\pm 5 \mathrm{MPa}$.

The welding conditions are shown in Table 1. In order to make martensite or ferrite in weld metal after welding, the welding conditions were estimated using the continuous cooling transformation (CCT) diagram for SM490 [9], as shown in Fig. 2, and the temperature history was estimated by a welding simulation [10]. We chose 50 and $100 \mathrm{~A}$ for the welding currents, and the specimens were named specimens A and $\mathrm{B}$, respectively. For the other welding conditions, the welding speed was $1.1 \mathrm{~mm} / \mathrm{s}$, the arc length was $3 \mathrm{~mm}$, the shielding gas was Ar, the shielding gas flow rate was $0.25 \mathrm{l} / \mathrm{s}$, the welding process was bead-on-plate by GTA welding, the electrode was pure tungsten with a diameter of $2.4 \mathrm{~mm}$, and the electrode extension was $3 \mathrm{~mm}$.

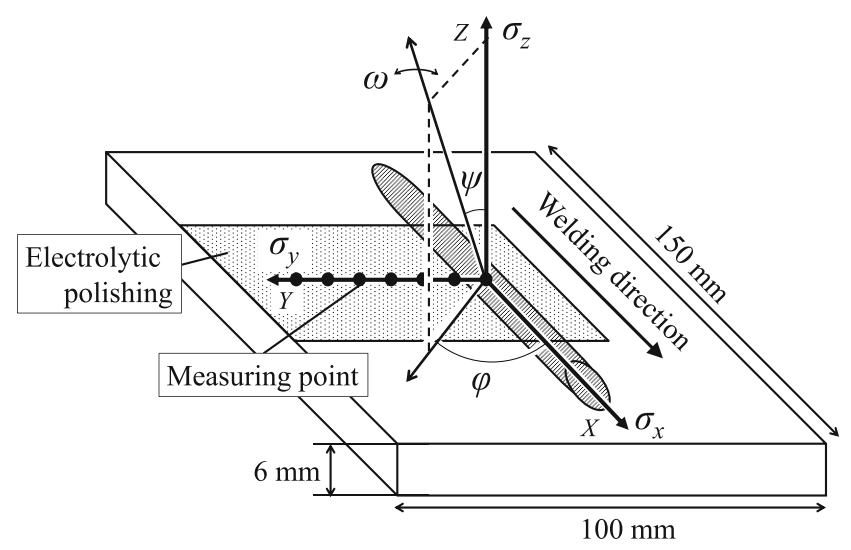

Fig. 1 Schematic illustration of specimen
Table 1 Welding conditions

\begin{tabular}{lll}
\hline & \multicolumn{2}{l}{ Specimen } \\
\cline { 2 - 3 } & $\mathrm{A}$ & $\mathrm{B}$ \\
\hline Welding current $1(\mathrm{~A})$ & 50 & 100 \\
Welding speed v $(\mathrm{mm} / \mathrm{s})$ & 1.1 & 1.1 \\
Shielding gas & $\mathrm{Ar}$ & $\mathrm{Ar}$ \\
Shielding gas flow rate $(e / \mathrm{s})$ & 0.25 & 0.25 \\
\hline
\end{tabular}

\subsection{X-ray stress measurement}

$\mathrm{X}$-ray stress measurement is a technique for measuring the strain between the diffraction plane spacings by X-ray diffraction, and several stress models have been proposed. Although X-ray stress measurement typically requires non-strained diffraction plane spacing $\left(d_{0}\right)$, welds are composed of various microstructures. Therefore, the measurement must consider their effects. In this study, we used the $2 \theta-\sin ^{2} \psi$ method [11], which does not require a precise $d_{0}$. The measuring conditions are shown in Table 2. The apparatus for X-ray stress measurement was AutoMATE (Rigaku). The diffraction peak was detected with a position-sensitive proportional counter (PSPC), and the diffraction peak was $\{211\}$ by $\mathrm{Cr}-\mathrm{K} \alpha$. The irradiation area of the specimen surface was limited by a collimator with a diameter of $1 \mathrm{~mm}$.

Since the diffraction plane strain measured by X-ray diffraction is different from the mechanical strain, the stress analysis must consider the dependence on the diffraction plane. That is, the X-ray elastic constants need to be estimated. From the elastic compliances for a single crystal, the X-ray elastic constants can be estimated using the Kröner model [12]. Since the irradiated region of the

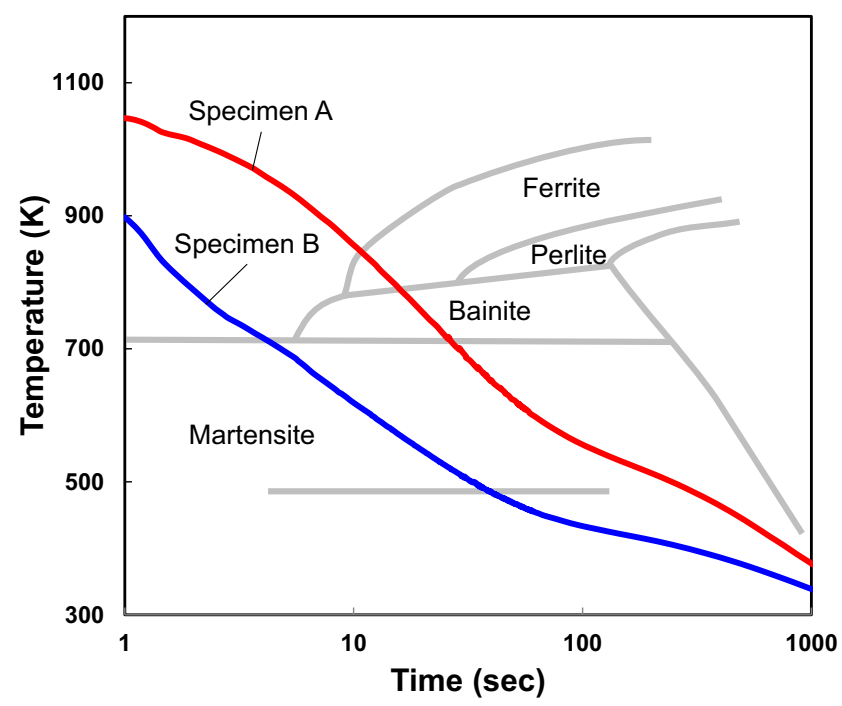

Fig. 2 CCT Diagram of SM490 [9, 10] 
Table 2 Measurement conditions

\begin{tabular}{ll}
\hline Apparatus & AutoMATE (Rigaku) \\
\hline Wavelength & $0.229100 \mathrm{~nm}(\mathrm{Cr}-\mathrm{Ka})$ \\
Tube voltage and current & $40 \mathrm{kv} 40 \mathrm{~mA}$ \\
Diffraction plane & $\{211\}$ \\
Collimator & $1 \mathrm{~mm}$ in diameter \\
Analysis model & $2 \theta-\sin ^{2} \psi$ model \\
\hline
\end{tabular}

$\mathrm{X}$-ray was limited, the crystal grains that contributed to $\mathrm{X}$-ray diffraction in the irradiated region decreased, and there was concern about a decrease in measurement accuracy, especially in the weld. Therefore, multi-axis oscillating, which had $\omega$ and $X$ oscillating parallel to the weld line, was performed in order to increase the crystal grain contribution to the X-ray diffraction, and the $\omega$ angle and the $X$ direction are defined as shown Fig. 1. As a result, in the weld metal and the heat-affected zone, the error in measurement was at most $\pm 30 \mathrm{MPa}$, and we were able to measure the residual stress with high precision.

The residual stress is determined using Eqs. (1), (2), and (3). The X-ray elastic constant $S_{1}$ and $S_{2}$ is indicated in Eqs. (4) and (5), respectively, and it is constructed using the $\mathrm{X}$-ray Young's modulus and X-ray Poisson's ratio considering the diffracting plane dependence.

$\sigma=K \cdot M$

$K=-\frac{\cot \theta_{0}}{2 S_{2}} \cdot \frac{\pi}{180}$

$M=\frac{\partial(2 \theta)}{\partial\left(\sin ^{2} \psi\right)}$

$S_{1}=-\frac{\nu_{h k l}}{E_{h k l}}$

$S_{2}=\frac{2\left(1+\nu_{h k l}\right)}{E_{h k l}}$

$\Sigma \quad$ Stress

$K \quad$ Stress constant

$M \quad$ Inclination of the $2 \theta-\sin ^{2} \psi$ diagram

$\mathrm{S}_{2} \quad$ X-ray elastic constant

$\theta_{0} \quad$ Non-strained diffraction angle $\psi \quad$ Normal of diffraction plane

$E_{h k l} \quad$ X-ray Young's modulus

$\nu_{h k l} \quad \mathrm{X}$-ray Poisson's ratio

\subsection{Microstructure observation}

The Vickers hardness distribution from the weld center to the base metal is shown in Fig. 3, and the microstructures observed by an optical microscope are shown in Figs. 4 and 5 . Both Vickers hardness distributions showed a maximum in the weld metal (the specimen A: $0-1.7 \mathrm{~mm}$, the specimen B: 0-3.8 $\mathrm{mm}$ ), and the maximum value in the specimen A was larger than that in the specimen B. From the results of the Vickers hardness test and microstructure observation, we distinguished that the weld metal in the specimen A had martensite, and that in the specimen B had bainitic ferrite, which means that the two specimens had different microstructures in the weld metal. Moreover, each Vickers hardness distribution showed almost the same. Therefore, homogeneity microstructure was made in each weld metal.

\subsection{Residual stress distribution}

The residual stress distributions are shown in Fig. 6a, b. Here, the heat-affected zone (HAZ; the specimen A: $y=1.7-3.0 \mathrm{~mm}$, the specimen B: $y=3.8-6.5 \mathrm{~mm}$ ) was defined as the area where the microstructure changed after welding without the weld metal (WM; the specimen A: $y=0-1.7 \mathrm{~mm}$, the specimen B: $y=0-3.8 \mathrm{~mm}$ ). The two specimens showed different residual stress distributions, respectively. In conventional behavior, it is known that the range of residual stress in the longitudinal direction $\left(\sigma_{x}\right)$ (which is tensile stress) depends on the heat input, and in the specimen B, for a large weld heat input, the range was larger than that of the specimen A. Also,

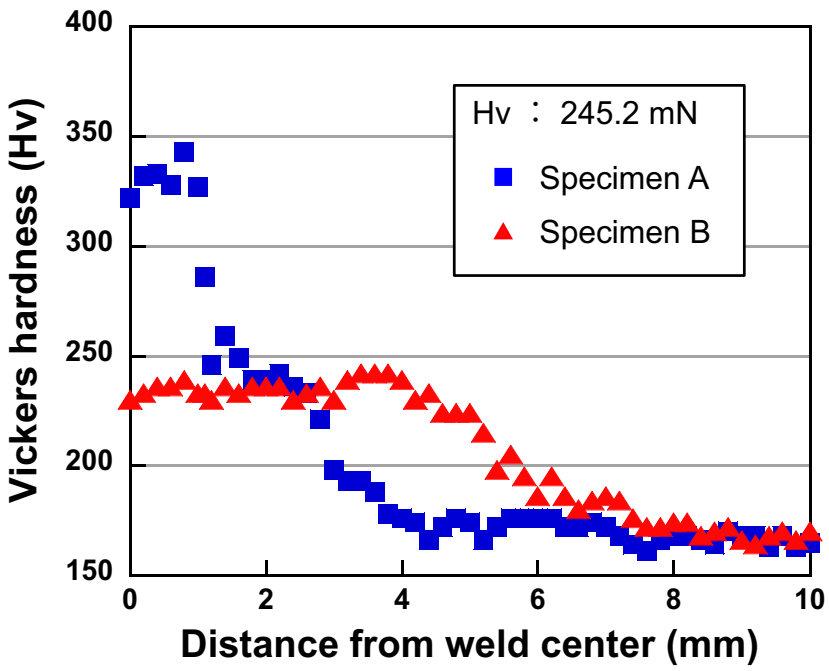

Fig. 3 Vickers hardness distribution in each specimen 


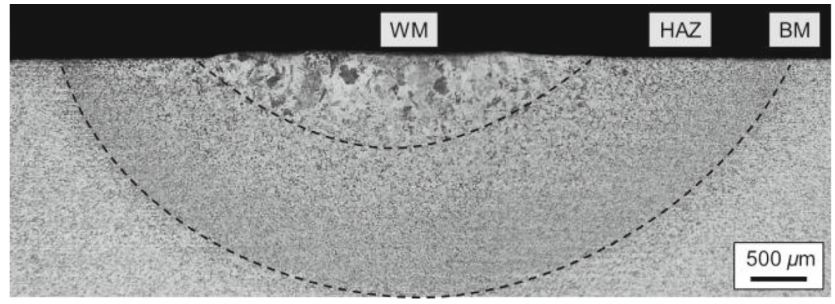

(a) Specimen A

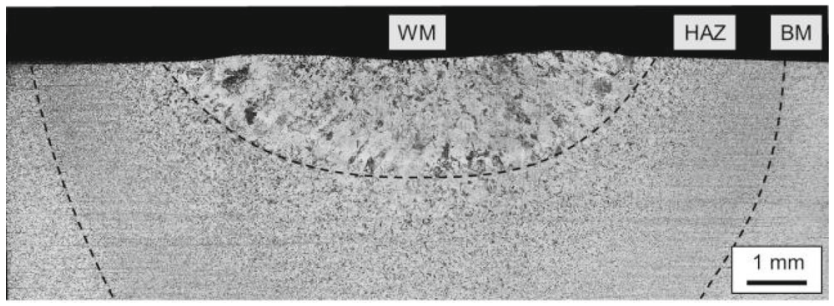

(b) Specimen B

Fig. 4 Macrostructure of weld in each specimen. a Specimen A; b specimen B

it is known that $\sigma_{x}$ in the center of the weld line typically depends on the yield stress of the material. The specimen A has martensite in the weld metal which has a high yield stress; however, $\sigma_{x}$ was smaller than that of the specimen B. It was considered that the tensile stress was reduced by expansion due to martensitic transformation. It appears that these results follow the conventional behavior regarding the residual stress distribution accompanied by phase transformation.

On the other hand, it is known that the residual stress in the transverse direction $\left(\sigma_{y}\right)$ at the center of the weld is about one-half the tensile yield stress. However, neither specimen followed this behavior. In the specimen A, it was considered that $\sigma_{y}$ changed to compression stress by expansion due to martensitic transformation. On the other hand, in the specimen $\mathrm{B}, \sigma_{y}$ was almost the same as $\sigma_{x}$, which had almost the same value as the yield stress; in the weld metal, the residual stress was an equal tensile stress in both axes. This result did not follow the conventional behavior.

As stated above, the residual stress distribution was different in each phase transformation behavior, and in some cases, the result of the X-ray stress measurement showed a characteristic tendency that did not necessarily follow the conventional behavior. One of the reasons why the result of the X-ray stress measurement did not follow the conventional behavior might be that we used the estimated X-ray elastic constant using the Kröner model. The weld is affected by various materials science factors, for example phase transformation, and it is not necessarily appropriate to use an elastic constant that is estimated by idealized models. Therefore, we measured the $\mathrm{X}$-ray elastic constant in the weld metal and reevaluated the residual stress distribution, and the effect on the characteristic stress distribution is discussed in the following chapter.

\section{Measurement of X-ray elastic constant and reevaluated residual stress}

\subsection{Material and four-point bending test conditions}

To measure the X-ray elastic constant in the specimens A, B, and base metal, three types of specimens for four-point bending test was made. As shown in Fig. 7, the specimens were thin plates with a size of $10 \mathrm{~mm} \times 60 \mathrm{~mm} \times 2 \mathrm{~mm}$ and were made by electrical discharge machining. As shown in Fig. 8, a strain gauge was attached at the center of the specimen, and the specimen was loaded by a four-point bending test machine. The load stress was increased in $50 \mathrm{MPa}$ increments from 0 to $400 \mathrm{MPa}$, and using the technique presented by Tanaka [13], the X-ray elastic constant $S_{1}$ and $S_{2}$ of the $\{211\}$ diffraction plane were determined from the intercept $\theta_{\psi=0}$ and inclination $M$ of the $2 \theta-\sin ^{2} \psi$ diagram, as shown in Eqs. (6) and (7). The load stress was calculated by multiplying the load strain and the mechanical Young's modulus $(2.05 \times$
Fig. 5 Microstructure of weld metal in each specimen. a Specimen A; b specimen B

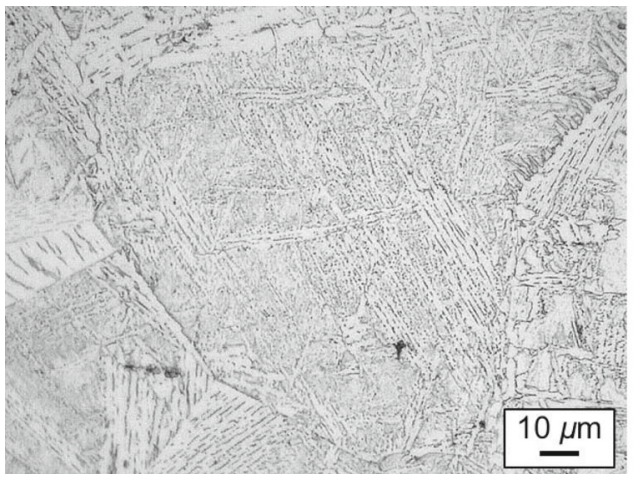

(a) Specimen A

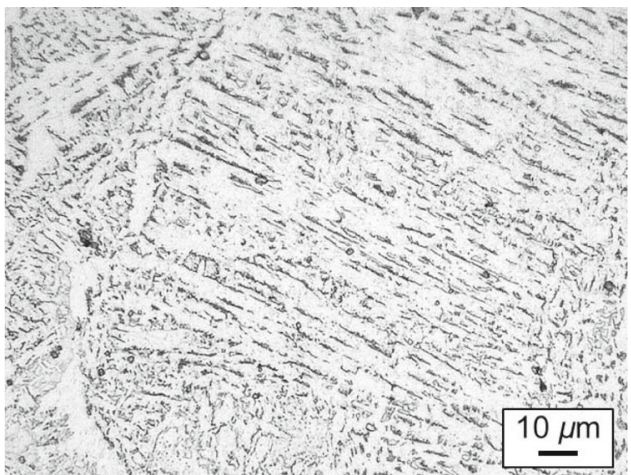

(b) Specimen B 
Fig. 6 Residual stress distribution in each specimen. a Specimen A; b specimen B

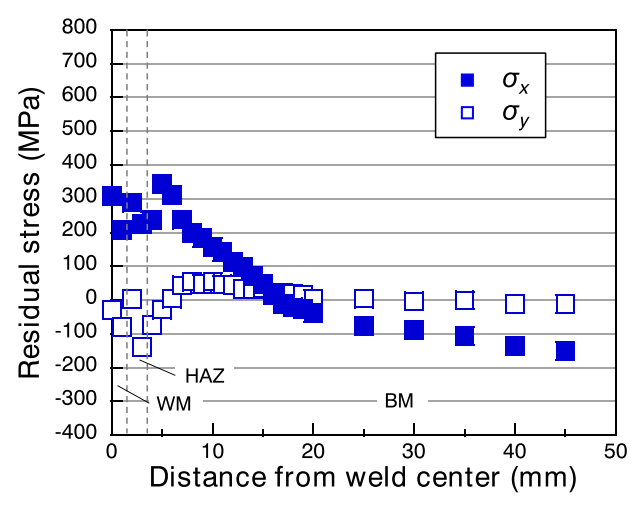

(a) Specimen A

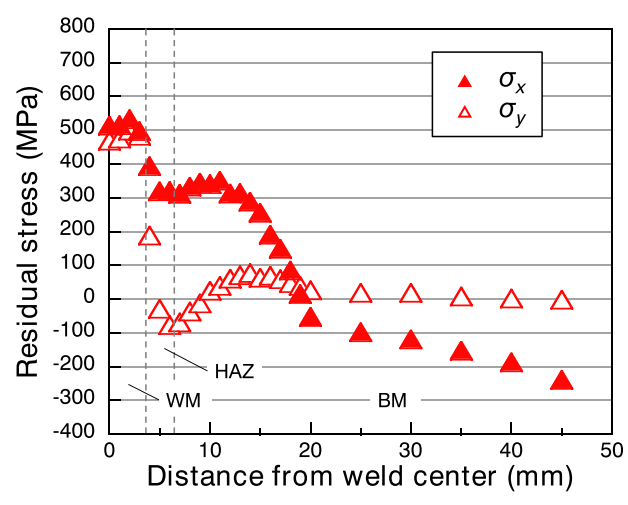

(b) Specimen B $\left.10^{2} \mathrm{GPa}\right)$. Then, X-ray Young's modulus $\left(E_{h k l}\right)$ and the X-ray Poisson's ratio $\left(\nu_{h k l}\right)$ are calculated from Eqs. (4), (5), (6), and (7), respectively.

$S_{1}=-\frac{\cot \theta_{0}}{2} \cdot \frac{\partial 2 \theta_{\psi=0}}{\partial \sigma_{A}} \cdot \frac{\pi}{180}$

$S_{2}=-2 \cot \theta_{0} \cdot \frac{\partial M}{\partial \sigma_{A}} \cdot \frac{\pi}{180}$

$S_{1}, S_{2} \quad$ X-ray elastic constant

$\theta_{\psi=0} \quad$ Intercept of the $2 \theta-\sin ^{2} \psi$ diagram

$M \quad$ Inclination of the $2 \theta-\sin ^{2} \psi$ diagram

$E_{h k l} \quad$ X-ray Young's modulus

$\nu_{h k l} \quad \mathrm{X}$-ray Poisson's ratio

$\theta_{0} \quad$ Non-strained diffraction angle

$\sigma_{A} \quad$ Load stress

3.2 Evaluation of X-ray elastic constant in the weld metal

The measured X-ray elastic constant, the X-ray elastic constant estimated by the Kröner model, and the stress constant $K$

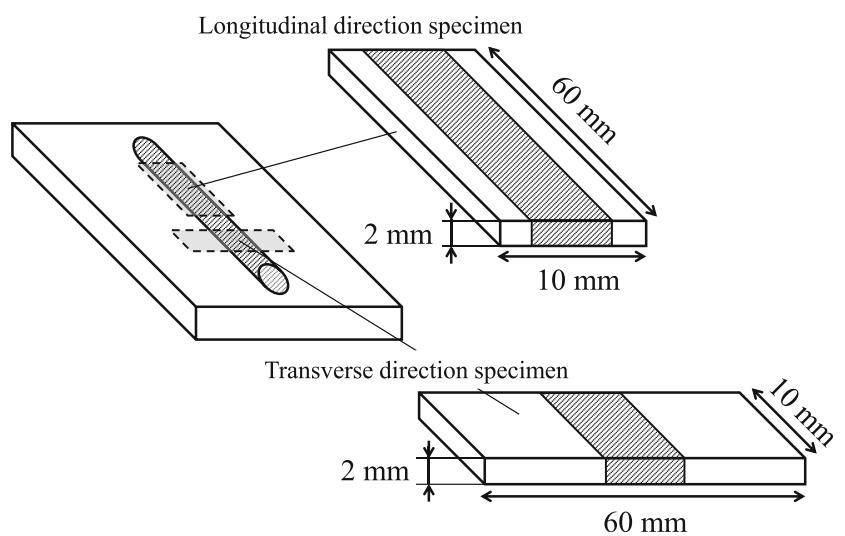

Fig. 7 Schematic illustration of specimen for four-point bending test on specimens A and B are shown in Table 3. In the specimen A, which had martensite in the weld metal, the calculated X-ray Young's modulus $\left(E_{h k l}\right)$ and X-ray Poisson's ratio $\left(\nu_{h k l}\right)$ in the longitudinal and transverse directions were almost the same values, and the value was almost the same as the value estimated by the Kröner model. Also, in base metal, the calculated X-ray Young's modulus and X-ray Poisson's ratio were almost the same as the value estimated by the Kröner model. On the other hand, in the specimen B, which had bainitic ferrite, the X-ray Young's modulus and the X-ray Poisson's ratio in the longitudinal and transverse directions were not the same value and showed anisotropy, and the value in the longitudinal direction was larger than the value estimated by the Kröner model.

\subsection{Reevaluation of residual stress using measured X-ray elastic constant}

The reevaluated residual stress distribution in the vicinity of the weld metal obtained using the measured X-ray elastic constant is shown in Fig. 9a, b. In the specimen

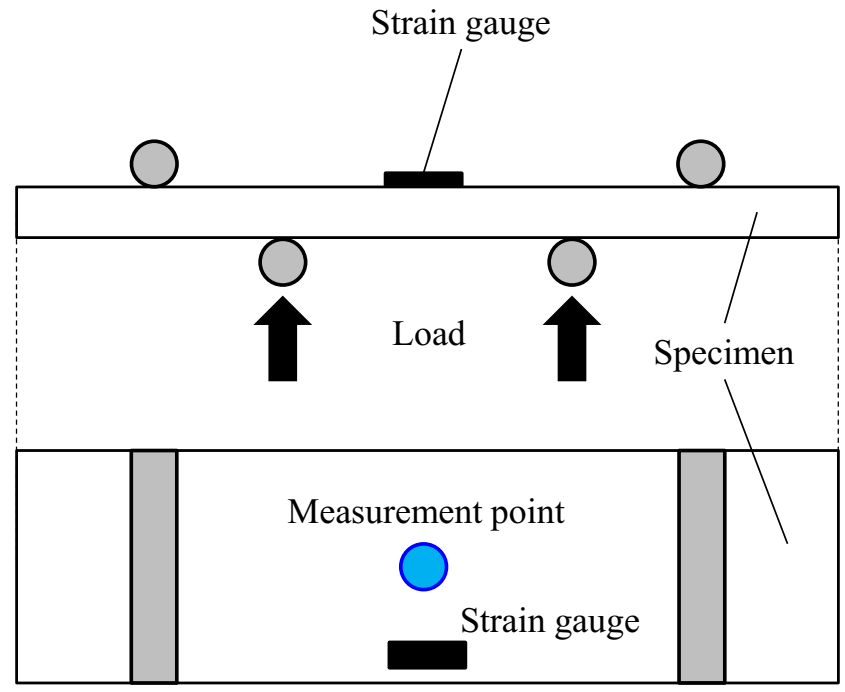

Fig. 8 Schematic illustration of four-point bending test 
Table 3 Measured and estimated $\mathrm{X}$-ray elastic constants

\begin{tabular}{llllll}
\hline & $S_{1}$ & $S_{2}$ & $E_{\text {hld }}(\mathrm{GPa})$ & $v_{\text {hld }}$ & $K$ \\
\hline Specimen A longitudinal direction & $-1.2810^{-6}$ & $1.1610^{-5}$ & 221.84 & 0.29 & -320.22 \\
Specimen A transverse direction & $-1.4810^{-6}$ & $1.2410^{-5}$ & 212.91 & 0.32 & -300.27 \\
Specimen B longitudinal direction & $-1.2610^{-6}$ & $0.9210^{-5}$ & 299.32 & 0.38 & -403.55 \\
Specimen B transverse direction & $-1.4110^{-6}$ & $1.2010^{-5}$ & 219.32 & 0.31 & -310.92 \\
Base metal & $-1.1910^{-6}$ & $1.1110^{-5}$ & 231.33 & 0.28 & -330.59 \\
Kroner model & $-1.2610^{-6}$ & $1.1410^{-5}$ & 224.70 & 0.28 & -324.69 \\
\hline
\end{tabular}

A, the X-ray elastic constant was almost the same as the value estimated by the Kröner model; therefore, the residual stress distribution barely changed. On the other hand, in the specimen B, the X-ray elastic constant showed anisotropy, and the value in the longitudinal direction was not the same as the value estimated by the Kröner model; therefore, $\sigma_{x}$ increased, and $\sigma_{y}$ barely changed. That is, in the specimen $\mathrm{B}$, it is thought that when we used the X-ray elastic constant estimated by the Kröner model, $\sigma_{x}$ in the weld metal was underestimated; however, when we used the measured X-ray elastic constant, we were able to evaluate $\sigma_{x}$ more accurately. Moreover, in the specimen B weld metal, since transverse direction stress $\sigma_{y}$ became large and it changed into the multi-shaft stress state, tolerance level of $\sigma_{x}$ to reach yielding must have become larger. Therefore, it is thought that the large $\sigma_{x}$ was appropriate in general.

In conclusion, it was shown that depending on the phase transformation behavior, the X-ray elastic constant in the weld metal has anisotropy, and it is difficult to use the X-ray elastic constant estimated by the Kröner model. However, if we use the measured value, we are able to evaluate the residual stress more accurately.

\section{Conclusion}

In this study, we used specimens with different microstructures in weld and evaluated the residual stress distribution by
X-ray stress measurement, focusing on the effect of phase transformation on the residual stress distribution.

In the specimen with martensite in the weld metal, a reduction in tensile stress occurred due to martensite transformation, and the X-ray stress measurement result followed the conventional behavior. On the other hand, in the specimen with bainitic ferrite in the weld metal, $\sigma_{y}$ was almost the same as $\sigma_{x}$, and the X-ray stress measurement result did not follow the conventional behavior.

When measuring the X-ray elastic constant in the specimen A, which had martensite in the weld metal, the X-ray elastic constant was almost the same as the value estimated by the Kröner model. On the other hand, in the specimen B, which had bainitic ferrite in the weld metal, the X-ray elastic constant showed anisotropy, and the value in the longitudinal direction was larger than the value estimated by the Kröner model.

Reevaluating the residual stress using the measured X-ray elastic constant, in the specimen A, in which the X-ray elastic constant did not show anisotropy, the residual stress distribution barely changed. On the other hand, in the specimen B, in which the X-ray elastic constant showed anisotropy, $\sigma_{x}$ increased, and $\sigma_{y}$ barely changed.

Depending on the phase transformation behavior, the X-ray elastic constant in the weld metal has anisotropy, and it is difficult to use estimated X-ray elastic constant by model; however, if we use the measured value, we are able to evaluate the residual stress more accurately.
Fig. 9 Reevaluated residual stress distribution in each specimen. a Specimen A; b specimen $B$

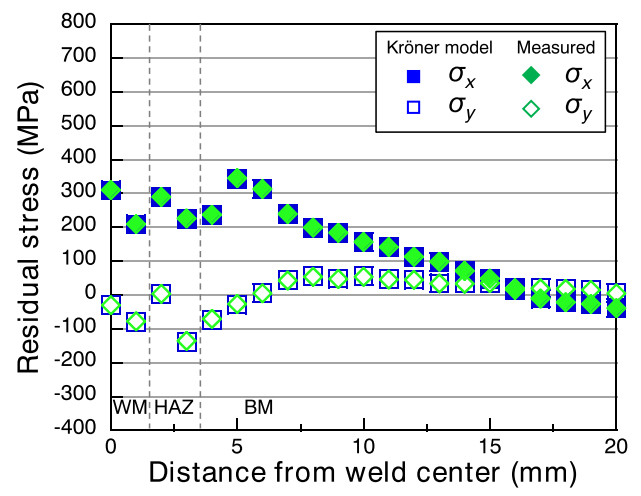

(a) Specimen $\mathrm{A}$

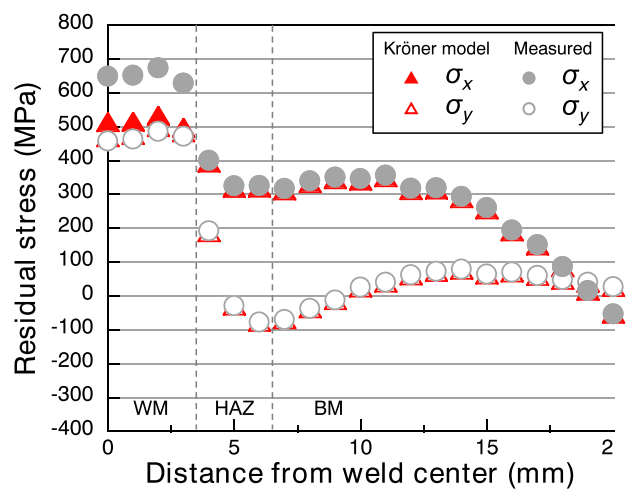

(b) Specimen B 
Open Access This article is distributed under the terms of the Creative Commons Attribution License which permits any use, distribution, and reproduction in any medium, provided the original author(s) and the source are credited.

\section{References}

1. Todoroki A, Kobayashi H (1986) Prediction of fatigue crack growth rate in residual stress field (application of superposition technique). Trans Japan Soc Mech Eng A 54:30-37

2. K. Nakacho, T, Ohta, N. Ogawa, S. Yoda, M. Sogabe and K. Ogawa, "Measurement of welding residual stresses by inherent strain method: New theory for axial-symmetry and application for pipe joint", Quarterly Journal of the Japan Welding Society, Vol. 27, No. 1 (2009) pp. 104-113

3. Mathar J (1934) Determination of initial stresses by measuring of the deformation around drilled holes. Trans Am Soc Mech Eng 56:249-254

4. Miyoshi Y (1989) X-ray analysis of residual stress in surface layer. J Japan Soc Prec Eng 55(8):1355-1359

5. Hashimoto T, Osawa Y, Hirano S, Mochizuki M, Nishimoto K (2011) Accuracy improvement of X-ray residual stress. Sci Technol Weld Join 16(3):261-266
6. Akita K, Yoshioka Y, Sano Y, Ogawa K, Kubo T, Obata M, Tanaka H (2005) X-ray residual stress measurement on weld metal of nickel based alloy. J Soc Mat Sci 54(7):710-716

7. Mochizuki M, Matsushima S, Kubo Y, Toyoda M (2005) Study on residual stress reduction by using phase transformation phenomena in welding material. J Japan Weld Soc 23(1):112-121

8. Murata H, Katoh N, Tamura H (1993) Effect of transformation on residual stress in welding-stress releasement by transformation superplasticity (part 5). Quart J Japan Weld Soc 11(4):545-550

9. NIMS, CCT diagram database

10. Okano S, Tanaka M, Mochizuki M (2011) Arc physics based heat source modelling for numerical simulation of weld residual stress and distortion. Sci Technol Weld Join 16(3):209-214

11. JSMS committee on X-ray study on mechanical behavior of materials, "Standard method for X-ray stress measurement - steel," JSMSSD-5-02 (2002) pp. 5-16

12. Kröner E (1958) Berechung der elastischen konstanten des vierkristalls aus den konstanten des einkristalls. Zeiteschrift Phys 151:504-518

13. Tanaka K, Kurimura T, Matsui E, Akiniwa Y (1987) X-ray measurement of residual stresses in sintered silicon nitride. J Soc Mat Sci 36: $817-822$ 